\title{
MICROTESTING AND CRYSTAL PLASTICITY MODELLING OF IN718 SUPERALLOY GRAINS
}

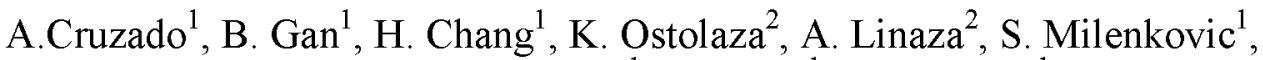 \\ J. M. Molina-Aldareguia ${ }^{1}$, J. Llorca ${ }^{1}$, J. Segurado $^{1}$ \\ ${ }^{1}$ IMDEA Materials Institute; C/Eric Kandel 2; Getafe, Madrid, 28906, Spain \\ ${ }^{2}$ Industria de Turbo Propulsores, ITP AS; Technological center Zamudio; Zamudio, Bizkaia, \\ 48170, Spain
}

Keywords: IN718, Micro-compression, Crystal plasticity

\begin{abstract}
A phenomenological crystal plasticity (CP) model is proposed to simulate the elasto-plastic behaviour of IN718 grains. The parameters of the CP model were fitted from micro-compression tests of a wrought IN718 alloy. A polycrystalline sample of IN718 was used for this investigation with an average grain size of $150 \mu \mathrm{m}$. Microstructural investigation revealed the sample was mainly composed of $\gamma$ phase, with negligible contents $(<1 \mathrm{vol} . \%)$ of $\delta$ phase particles at the grain boundaries and $\mathrm{MC}$ carbides. The micro-compression tests were carried out in singlecrystal orientations, by machining single-crystal micropillars inside individual grains using a focused ion beam (FIB) system and compressing them using a flat punch inside an instrumented nanoindentation system. Different micropillar compression tests were performed to quantify the effects of pillar diameter, strain rate and pillar orientation. A finite element (FE) model of the micropillar was used to fit the $\mathrm{CP}$ model with the experimental results. The calibration of the $\mathrm{CP}$ model was done by considering only the plastic contribution of the experimental stress-strain curves, avoiding the initial tip-micropillar misalignments and the local plastic flow prior to pillar yield. The resulting CP model was then evaluated by comparing the simulation of other independent micro-compression tests with the corresponding experimental result and the agreement was remarkable in all the cases. This model will be used as a basis to develop a polycristalline FE CP model of IN718.
\end{abstract}

\section{Introduction}

IN718 is an Iron-Nickel based Superalloy strengthened with $\gamma^{\prime}$ and $\gamma^{\prime \prime}$ precipitates for structural applications in engines up to $650-700^{\circ} \mathrm{C}$. It combines good corrosion resistance and high mechanical properties with excellent weldability [1]. The strength of this alloy comes from coherent precipitates, which are of a small part $\gamma^{\prime}-\mathrm{Ni}_{3}(\mathrm{Al}, \mathrm{Ti})$ but mostly $\gamma^{\prime \prime}-\mathrm{Ni}_{3} \mathrm{Nb}$ [2]. Both of these precipitates are found in the $\gamma \mathrm{Ni}$ rich matrix (face centered cubic-A1). The ordered $\gamma^{\prime}$ phase presents the $\mathrm{Ll}_{2}$ crystal structure and the $\gamma^{\prime \prime}$ is body centred tetragonal (BCT) corresponding to the $\mathrm{D}_{22}$ structure [3]. The $\gamma^{\prime \prime}$ phase is a metastable form of $\mathrm{Ni}_{3} \mathrm{Nb}$ which tends to stabilize to orthorhombic $\delta$ phase with the structure of $\mathrm{D} 0_{\mathrm{a}}[4]$.

In general, IN718 alloys present a marked tension-compression strength differential [5], softening under cyclic loading conditions [6] and significant losses of strength at temperatures above $650^{\circ} \mathrm{C}$. Plastic behavior depends strongly on microstructure, with the most important microstructural parameters being grain size [7], and the $\gamma^{\prime}$ and $\gamma^{\prime \prime}$ precipitate size and volume 
fraction [8]. Therefore, experimental characterization of the mechanical properties of IN718 as a function of microstructure requires a large number of tests that are both costly and time consumming. To overcome this problem, the objective of this work is to develop a microstructure-based numerical model capable of predicting the mechanical behavior of polycrystalline IN718.

A state of art approach for developing models of the mechanical behaviour based on the microstructure is the use of crystal plasticity (CP) to predict grain behaviour. In this case, the overall polycrystalline behavior is obtained by numerical homogenization (normally by using FEM) over a volume element that includes the real microstructural features such as grain size, shape and orientation distribution $[11,12]$. The accuracy of the polycrystalline model will strongly depend on a correct modelling of the grain behavior, able to reproduce the main deformation mechanisms of that particular alloy.

The present paper aims to develop and fit a crystal plasticity model able to predict the mechanical behavior on the single crystalline scale. This model will be the basis of following works to develop a polycrystalline model. Although the behavior of the grains also depends on their particular microstructure (precipitate contents, shape and distribution), in this study, only one material will be considered, characteristic of a wrought alloy with coarse grains. The behavior of the grain will be obtained by compression of micropillars machined by Focused Ion Beam (FIB) on crystals with different orientations, performed at different strain rates and with different micropillar sizes.

\section{Experimental characterization}

\section{Microstructure characterization}

The volume fraction and spatial distribution of the different phases within the grains were studied in a generic polycristalline IN718 microstructure with coarse grains (ASTM 2.5) as shown in Figure 1a. The grains were analyzed by scanning electron microscopy (SEM) in order to determine the different phases $\left(\gamma\right.$ matrix, $\gamma^{\prime}$ and $\left.\gamma^{\prime \prime}\right)$ as well as the shape, volume fraction and spatial distribution of the MC carbides and $\delta$ phase particles.

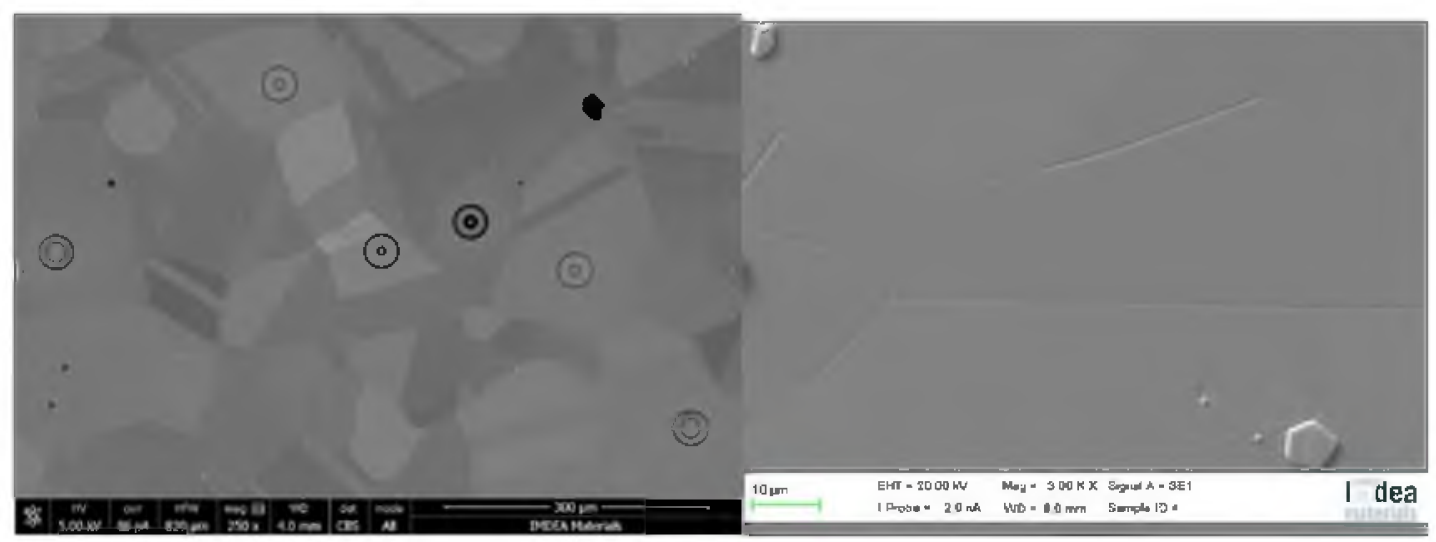

a)

b)

Figure 1. SEM micrograph of a wrought IN718 superalloy with ASTM 2.5 grains: a) microstructure with different grain orientations and micropillars b) magnification of the microstructure where $\mathrm{MC}$ carbides and $\delta$ phase precipitates are shown in a very low quantity. 
Microstructural investigation revealed the sample was mainly composed of $\gamma$ phase, with negligible contents $(<1 \mathrm{vol} . \%)$ of $\delta$ phase particles at the grain boundaries and dispersed MC carbides. No presence of $\gamma^{\prime}$ and $\gamma^{\prime \prime}$ phases was observed within the resolution of the SEM, but their size is expected to be an order of magnitude smaller than that of the MC carbides and $\delta$ phase particles and they are expected to be dispersed within the $\gamma$ phase. Therefore, the CP model developed in this work represents the behavior of the $\gamma$ phase grains, including the dispersed $\gamma^{\prime}$ and $\gamma^{\prime \prime}$ phases.

\section{Micromechanical characterization: Micro-Pillar compression}

The calibration of the CP model was carried out by direct micro-mechanical testing of the individual $\gamma$ phase grains. The micro-compression tests were carried out in single-crystal orientations, by machining single-crystal micropillars inside individual grains using a focused ion beam (FIB) system (FEI Helios 600i). The micropillar orientation was identified by electron backscatter diffraction (EBSD) using an Oxford AZTEC system. Figure 2 shows one of these micropillars, $5 \mu \mathrm{m}$ in diameter and $12 \mu \mathrm{m}$ in length, used for the micromechanical studies. The pillars were subsequently compressed using a diamond flat punch inside an instrumented nanoindentation system (Hysitron T1950). The compression tests were carried out in displacement control. Different tests were performed to quantify the effects of pillar diameter, strain rate and crystal orientation to incorporate those effects into the $\mathrm{CP}$ model.

The effect of pillar diameter was assessed by testing pillars with diameters ranging between 3 and $5 \mu \mathrm{m}$, maintaining a constant length to diameter ratio of 2.4 . The effect of strain rate was captured by performing tests with three different strain rates $\left(10^{-4}, 10^{-3}\right.$ and $\left.10^{-2} \mathrm{~s}^{-1}\right)$. All tests to assess the effect of pillar diameter and strain rate were performed in crystal orientations favorably oriented for single-slip. Finally, other pillar orientations, including those oriented for multiple slip, were also analyzed keeping a constant pillar diameter of $5 \mu \mathrm{m}$ at an applied strain rate of $10^{-3}$.

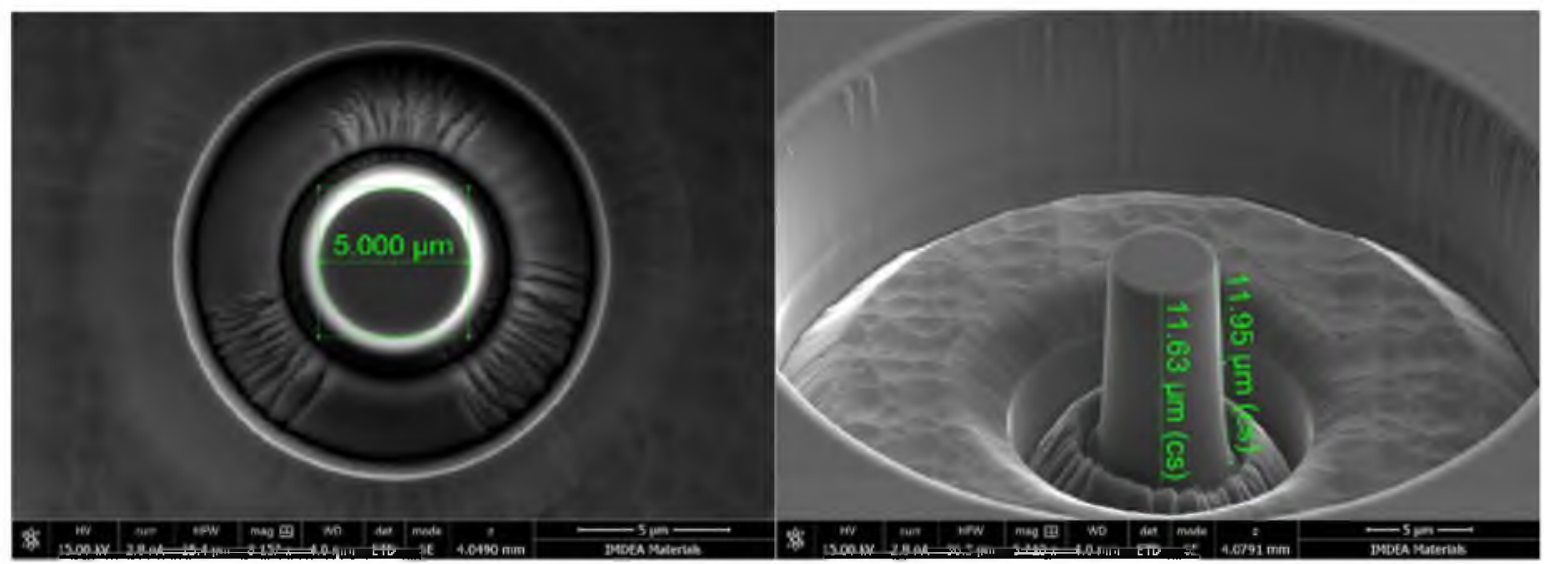

Figure 2. Micropillar machined inside a single grain: a) top view and b) viewed at an angle of $52^{\circ}$.

\section{Crystal plasticity model}

The crystal plasticity model is based on the multiplicative decomposition of the deformation gradient, $\boldsymbol{F}$, into its elastic $\boldsymbol{F}^{e}$ and plastic part $\boldsymbol{F}^{p}$ (1): 


$$
\boldsymbol{F}=\boldsymbol{F}^{e} \cdot \boldsymbol{F}^{p}
$$

The configuration defined by $\boldsymbol{F}^{p}$, which is formed due to the dislocation motion, is called the relaxed or intermediate configuration and is associated with a unrecoverable deformation preserving the crystal lattice orientation. The configuration defined by $\boldsymbol{F}^{e}$ models the reversible elastic stretch and the rigid body rotation of the lattice. Applying the definition of the velocity gradient, expression (1) leads to (2).

$$
\boldsymbol{L}=\dot{\boldsymbol{F}} \cdot \boldsymbol{F}^{-1}=\dot{\boldsymbol{F}}^{e} \boldsymbol{F}^{e^{-1}}+\boldsymbol{F}^{e} \dot{\boldsymbol{F}}^{p} \boldsymbol{F}^{p^{-1}} \boldsymbol{F}^{e^{-1}}
$$

If plastic deformation takes place along multiple slip systems, the plastic velocity gradient $\overline{\boldsymbol{L}}^{p}$ in the intermediate configuration is defined as the sum of the shearing rates $\dot{\gamma}^{\alpha}$, on all the slip systems $\alpha$, according to (3):

$$
\overline{\boldsymbol{L}}^{p}=\dot{\boldsymbol{F}}^{p} \cdot \boldsymbol{F}^{p^{-1}}=\sum_{\alpha=1}^{N_{s l i p}} \dot{\gamma}^{\alpha}\left(\boldsymbol{s}_{0}^{\alpha} \otimes \boldsymbol{m}_{0}^{\alpha}\right)
$$

The crystal was assumed to behave as an elasto-viscoplastic solid in which the plastic slip rate for a given slip plane system follows a power law dependency shown in (4):

$$
\dot{\gamma}^{\alpha}=\dot{\gamma}_{0}\left|\frac{\tau^{\alpha}}{\tau_{c}^{\alpha}}\right|^{1 / m} \operatorname{sgn}\left(\tau^{\alpha}\right)
$$

Here $\dot{\gamma}_{0}$ is the reference strain rate, $\tau_{c}^{\alpha}$ is the deformation resistance of $\alpha^{\text {th }}$ slip system and $m$ is the rate sensitivity parameter. $\tau^{\alpha}$ is the resolved shear stress on the slip plane $\alpha$, and is obtained as the projection of the Kirchoff stress on the slip system (5),

$$
\tau^{\alpha}=\mathbf{C}\left[\frac{1}{2}\left(\boldsymbol{F}^{e^{t}} \boldsymbol{F}^{e}\right)-\mathbf{I}\right]:\left(\mathbf{s}_{0}^{\alpha} \otimes \boldsymbol{m}_{0}^{\alpha}\right)
$$

where $\mathbf{C}$ represents the elastic stiffness tensor of the crystal.

The evolution of the critical resolved shear stress $\tau_{c}^{\alpha}$ of a given slip system $\alpha$, is obtained according to equation 6 ,

$$
\dot{\tau}_{c}^{\alpha}=\sum_{\beta} h_{\alpha \beta}\left|\dot{\gamma}^{(\beta)}\right|
$$

where $\beta$ stands for any slip system and $h_{\alpha \beta}$ is the strain hardening modulus due to self and latent hardening and is expressed in a simplify manner following the equation 7 :

$$
h_{\alpha \beta}=q_{\alpha \beta} h
$$


where $q_{\alpha \beta}$ are the interaction coefficients that represent the influence of the hardening between different slip systems, and $h$ corresponds to the self hardening modulus. The self hardening modulus $h$ is modeled according to the Asaro-Needleman [13] hardening law (8),

$$
h\left(\gamma_{a}\right)=h_{0} \sec h^{2}\left|\frac{h_{0} \gamma_{a}}{\tau_{s}-\tau_{0}}\right|
$$

where $h_{0}$ is the initial hardening modulus, $\tau_{0}$ the initial yield shear stress, $\tau_{s}$ the saturation yields shear stress and $\gamma_{a}$ is the accumulated shear strain in all slip systems given by equation 9

$$
\gamma_{a}=\int_{0}^{t} \sum_{\alpha} \dot{\gamma}^{\alpha} d t
$$

Hence, the aim of this work is to fit the different parameters involved in the previously described hardening law by using the microcompresion tests data in different single crystal orientations.

\section{Numerical analysis of micro-compression test rig}

\section{$\underline{\text { FEM model }}$}

The FE used for the simulation of micro-compression tests for different crystal orientations is shown in Figure. 3. The model is developed in Abaqus 6.10.1 and represents the same geometry used in the $5 \mu \mathrm{m}$ diameter test. Moreover the curvature radius in the fillet (approximately $\mathrm{r}=1.5 \mu \mathrm{m})$ and the tapper angle approximately $\beta=1.5^{\circ}$ was included.

The pillar and the supporting material were modeled as a single crystal. Eight nodes lineal brick elements (C3D8) were used for discretization and a mesh with 15359 elements that leads to converged results was used. The indenter 'tip' was modeled as a rigid body, with a lateral stiffness of $10 \mu \mathrm{N} / \mathrm{nm}$ which is approximately the lateral stiffness of the indenter used in the experiment. The tip-micropillar contact was modeled according to a Coulomb friction model, with a friction coefficient of 0.1 . While a displacement boundary condition was introduced in the vertical direction of the tip the base of the supporting material was fully constrained.

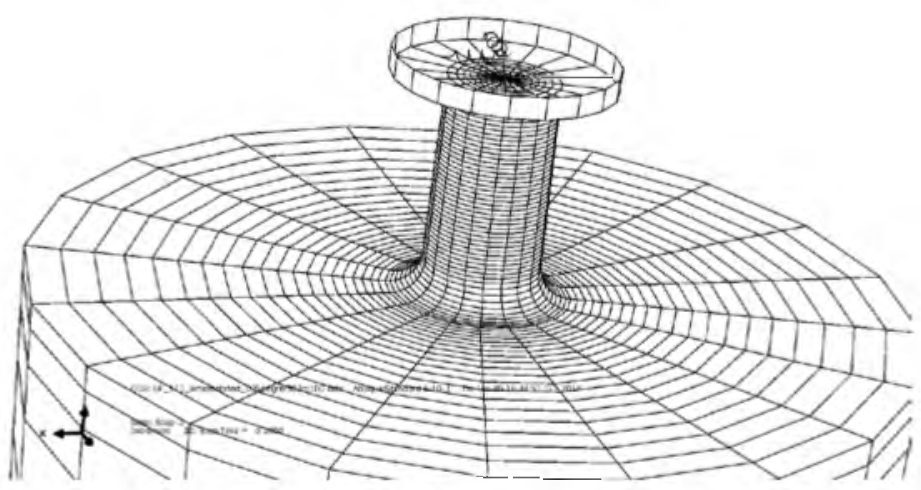

Figure 3. FEM model of the micropillar. 
The crystal behaviour was introduced following the crystal plasticity model presented previously and according to the FCC structure of the $\gamma$ phase the 12 slip systems corresponding to the octahedral family were introduced. The elastic constants of the cubic single crystal were taken from the literature [14] and can be found in Table I. The parameters that controls the plastic deformation of each slip system $\tau_{0}, \tau_{s} h_{0}$ and $q_{\alpha \beta}$ will be fitted according to the experimental results for different crystal orientations.

Table I. Elastic constants of the cubic single crystal in IN718 [14].

\begin{tabular}{|l|l|l|}
\hline $\mathrm{C}_{11}(\mathrm{GPa})$ & $\mathrm{C}_{12}(\mathrm{GPa})$ & $\mathrm{C}_{44}(\mathrm{GPa})$ \\
\hline 259.6 & 179 & 109.6 \\
\hline
\end{tabular}

\section{Crystal plasticity model fitting and evaluation}

In order to fit the viscoplasticity model and to minimize the possible errors due to the tipmicropillar misalignement, only the plastic contribution of the engineering stress-strain curves was considered for carrying out the fitting procedure.

The different parameters of the plasticity model were obtained following a specific strategy that will be detailed in the next paragraphs. Firstly the rate sensitivity exponent was fitted by linear regression of the natural logarithm of equation 3 as shown in equation 10. Taking the reference values of $\dot{\gamma}_{0}$ and $\tau_{c}^{\alpha}$ (saturated yield stress) the ones corresponding to a strain rate of $10^{-3} \mathrm{~s}^{-1}$, the fitting was carried out by linear regression of the points obtained with the saturated yield stress $\tau_{s}^{\alpha}$ corresponding to $10^{-4}$ and $10^{-2} \mathrm{~s}^{-1}$ strain rates. The linear regression shown in Figure 4 gives a strain rate sensitive exponent of $m=0.017$ considering the initial strain rate of $\dot{\gamma}_{0}=2.42 \cdot 10^{-3} \mathrm{~s}^{-1}$. It means that the behaviour of the analyzed material is close to rate independent.

$$
\ln \left(\frac{\dot{\gamma}^{\alpha}}{\dot{\gamma}_{0}}\right)=n \cdot \ln \left|\frac{\tau_{s}^{\alpha}}{\tau_{c}^{\alpha}}\right|
$$

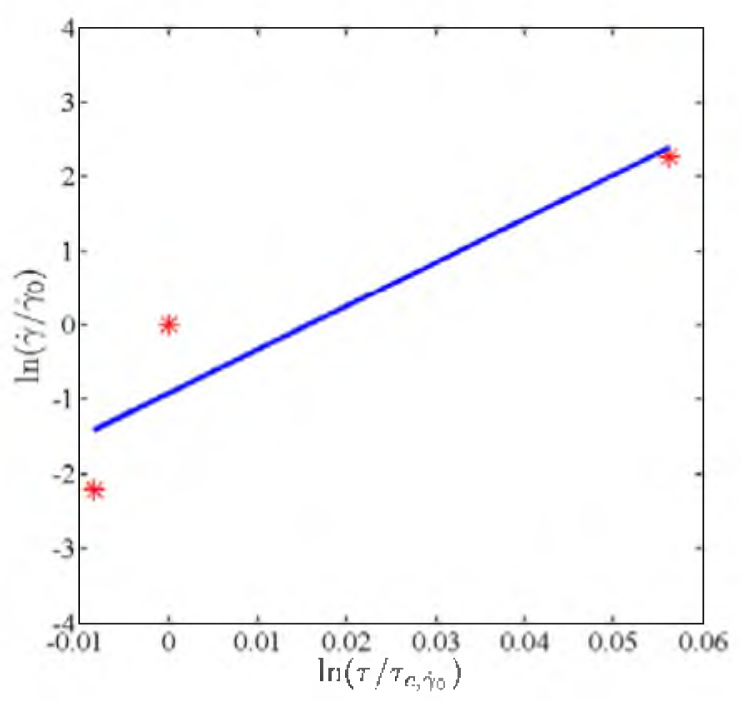

Figure 4. Strain rate sensitive exponent fitting. 
The second step of the specific strategy was to fit the parameters defining the self hardening, $\tau_{s}, \tau_{0}$ and $h_{0}$, with the resolved shear stress-engineering plastic strain curve obtained from a microcompression test oriented in single slip, as shown in figure 5a. The resulting values of the fitting normalized with respect to $\tau_{0}$ are shown in Table II. Furthermore figure 5a shows the small influence of the micropillar size in this diameter range.

Table II. Normalized values of the self hardening parameters.

\begin{tabular}{|c|c|}
\hline$\tau_{s} / \tau_{0}$ & $h_{0} / \tau_{0}$ \\
\hline 1.28 & 24.5 \\
\hline
\end{tabular}

Figure $5 \mathrm{~b}$ shows the prediction of the model, fitted with $\tau_{s}, \tau_{0} h_{0}$ and $m$, of the tests performed at different strain rates and it can be observed that the model captures relatively well the rate dependency behavior observed experimentally.

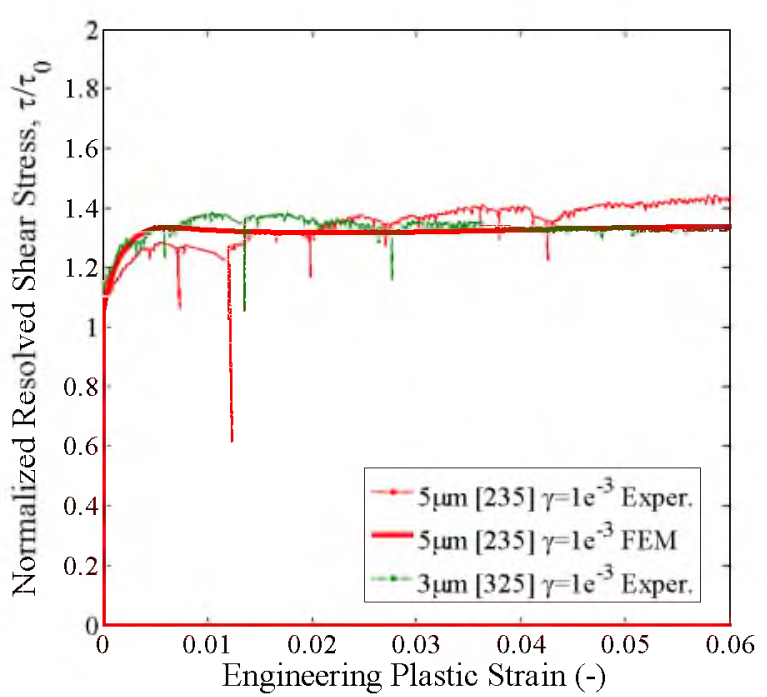

a)

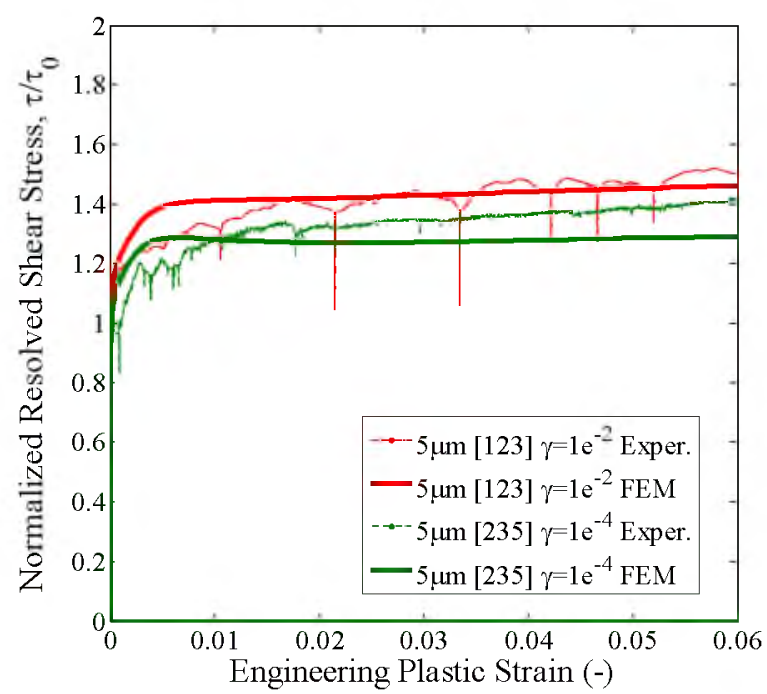

b)

Figure 5. CP model fitting in single sliding orientation with: a) $3 \mu \mathrm{m}-5 \mu \mathrm{m}$ diameter and $1 \mathrm{e}^{-3}$ strain rate and b) $5 \mu \mathrm{m}$ diameter and $1 \mathrm{e}^{-2}-1 \mathrm{e}^{-3}$ strain rates

The next step is to determine the latent hardening coefficients from the engineering stressengineering strain curves. In order to illustrate the effect of the latent hardening parameter, FE simulation of pillars oriented in a single slip [235] and a double slip [212] directions were performed using $\mathrm{q}$ values from 1 to 2 . It was observed (Fig 6a) that the response of pillars in single slip is unaffected by $q$ while in double slip the resolved stress increases in $15 \%$ from $q=1$ to $\mathrm{q}=2$. The experimental curves used to obtain this value are compression curves on pillars oriented in directions producing coplanar [212] and non coplanar [025] double slip, as shown in figure $6 \mathrm{~b}$. It was found that the value $\mathrm{q}=1$ (for both coplanar and non coplanar slip systems) produce the best agreement for both directions, indicating that this material follows an isotropic hardening model. 


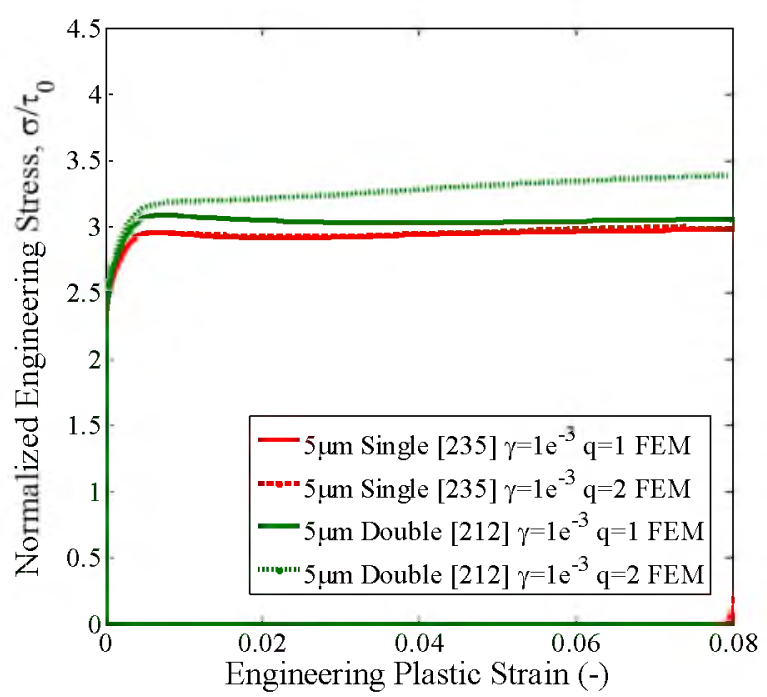

a)

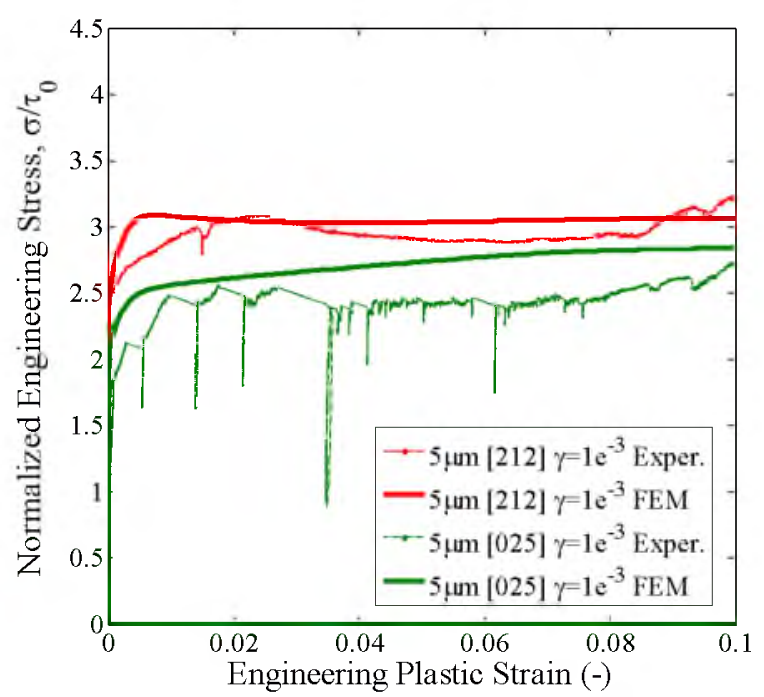

b)

Figure 6. CP model fitting in double sliding orientation: a) influence of latent hardening in a single and double slip orientation and b) experimental-FEM correlation with a latent hardening $\mathrm{q}=1$.

Finally the model was tested in specimens with single/double activated slip planes (it means that in this type of orientations there is a second Schmidt factor which is very close to the maximum Schmidt factor) and with multiple slip. In the first type of crystal orientations (Figure 7a) the model qualitatively reproduce the experimental behavior but cannot capture precisely the flow stress in [546] and overestimates the yield point in the [216] orientation. On the contrary, in the case of pillars oriented for multiple slip (Figure 7b) the model captured accurately the experimental behavior.

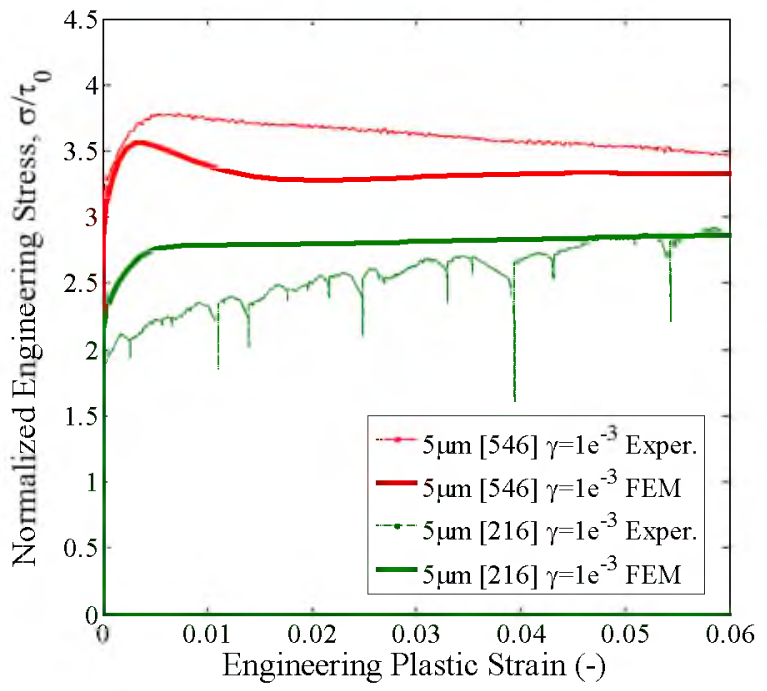

a)

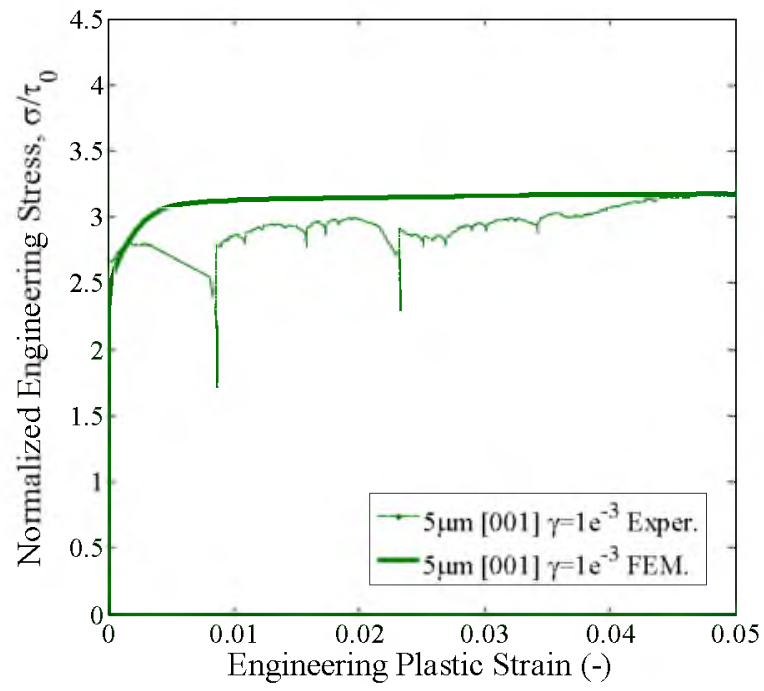

b)

Figure 7. CP model correlation with multiple orientations: a) single/double slip orientation and b) multiple slip orientation. 
Finally, in addition to the ability for predicting reasonably well the stress-strain behavior for any pillar orientation and strain rate (Figures $5 \mathrm{~b}$ and 7 ), the model is able to accurately reproduce the shape of the deformed pillar and the formation of a slip band. This is shown as an example for the specimen orientated in the [212] direction, where both coplanar slip systems are activated, in Figure 8 .

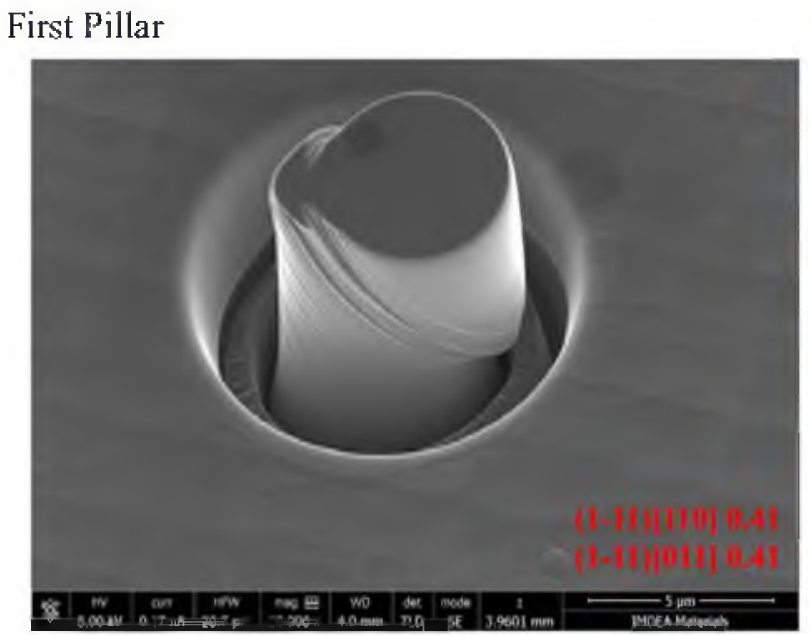

a)

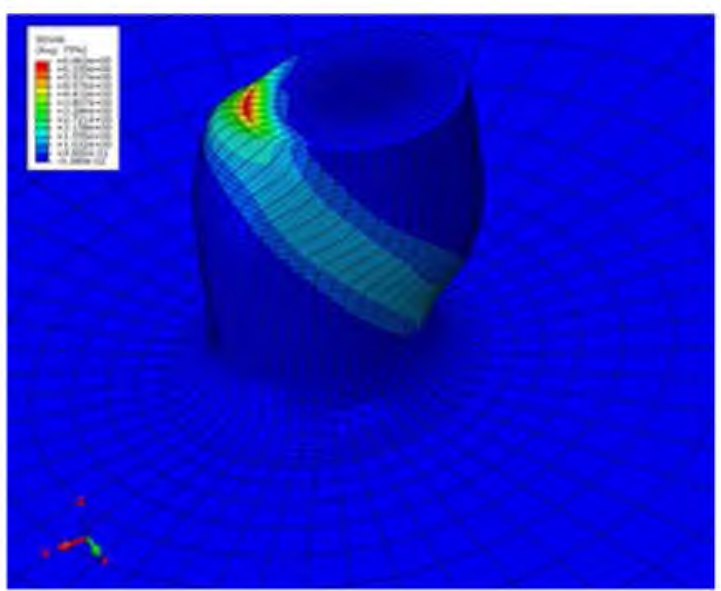

b)

Figure 8. Experimental-FEM correlation in a $5 \mu \mathrm{m}$ diameter- $1 \mathrm{e}^{-3}$ and [212] crystal orientation:

a) Experiemental micropillar capture, b) accumulated plastic strain plot of the FEM model.

\section{Conclusions}

A crystal plasticity (CP) model has been developed to predict the elastic-plastic behavior of IN718 grains. The parameters defining the CP behavior are obtained by testing in compression micro-pillars built by focused ion beam inside different grains.

The CP model was adjusted by comparison of a finite element (FE) model of the pillar compression test with the real experiments.

The compression tests included different pillar orientations, sizes and different applied strain rates. It was found that the effect of the pillar diameter was minimal in the range 3-5 microns and the strain rate sensitivity was very small.

The model parameters obtained using a selected set of experiments were the strain rate sensitivity parameter $\mathrm{m}=0.017$, the parameters defining the self-hardening function $\left(\tau_{s}, \tau_{0}\right.$ and $h_{0}$ ) and the latent-hardening coefficient, $\mathrm{q}=1$, indicating isotropic hardening. Finally, in order to check the accuracy of the adjusted model, independent tests with different orientations and strain rates were predicted and the agreement between numerical results and experiments was remarkable. In addition, the model was able to accurately predict the deformation of the pillar, including the formation of the slip bands observed in the experiments. 


\section{Acknowledgements}

The authors would like to thank the European Union for the financial support given under the Clean Sky Joint Undertaking, 7th Framework Programme, in the frame of the MICROMECH project (CS-GA-2013-620078).

\section{References}

1. H.J. Wagner, A.M. Hall, "Physical metallurgy of alloy 718”. DMIC Report 217, Battelle Memorial Institute, Columbus, June 1, 1965.

2. J. F. Radavich, "The Physical Metallurgy of Cast and Wrought Alloy 718", in Superalloy 718Metallurgy and Applications, E. A. Loria, Ed.,TMS, 229-240, 1989.

3. H.E. Jianjong, " $\gamma$ " Precipitate in Inconel 718," Journal of Material Science and Technology, 10 (1994), 293-303.

4. J. Dong, X. Xie, Z. Xu, S. Zhang, M. Chen, J. F. Radavich, TEM study on microstructure behaviour of alloy 718 after long time exposure at high temperatures, in Superalloys 718, 625, 706 and Various Derivatives 1994, E. A. Loria, Ed., TMS, 649-658, 1994.

5. S. K. Iyer, C. J. Lissenden, "Multiaxial constitutive model accounting for the strengthdiferential in Inconel 718," International Journal of plasticity, 19 (2003), 2055-2081.

6. D. Gustafsson, J.J. Moverare, K. Simonsson, S. Sjöström, "Modeling of the Constitutive behaviour of Inconel 718 at Intemediate temperatures," Journal of Engineering for Gas Turbines and Power, 133 (2011), 1-4.

7. Y.S.Song, M.R. Lee, J.T.Kim, Effect of grain size for the tensile strength and the low cycle fatigue at elevated temperature of alloy 718 cogged by open die forging press, in Superalloys 718, 625, 706 and Derivatives 2005, E. A. Loria, Ed., TMS, 539-549, 2005.

8. C. Ruiz, A.Obabueki, K. Gillespie, Evaluation of the microstructure and mechanical properties of delta processed alloy 718, in Superalloys 1992, S.D. Antolovich et al., Ed.,TMS, 33-42, 1992.

9. P.E. McHugh, R. Mohrmann, "Modelling of creep in a Ni base superalloy using a single crystal plasticity model”, Computational Materials Science, 9 (1997), 134-140.

10. E.N, Tedjaseputra, "Numerical simulation of microstructure-based crystal plasticity finite element model for titanium and nickel alloys" (Ph.D. thesis, The Ohio State University, 2012).

11. S. Keshavarz, S. Ghosh, "Multi-scale plasticity finit element model approach to modelling nickel-based superalloys", Acta Materiala, 61 (2013), 6549-6561.

12. M. Shenoy, J. Zhang, D. L. McDowell, "Estimating fatigue sensitivity to polycrystalline Nibase superalloy microstructures using a computational approach", Fatigue and fracture of enginering materials and structures, 30 (2007), 889-904. 
13. R.J. Asaro, A. Needleman, "Overview no. 42 Texture development and strain hardening in rate dependent polycrystals", Acta metallurgica, 33 (1985), 923-953.

14. G. Martin, N. Ochoa, K. Saï, E. Hervé-Luanco, G. Cailletaud, "A multiscale model for the elastoviscoplastic behavior of Directionally Solidified alloys: Application to FE structural computations", International Journal of Solids and Structures, 51 (2014), 1175-1187. 